\title{
OPTIMIZATION OF LONG-ENDURANCE AIRFOILS
}

\author{
J. Hua*, F.M. Kong ${ }^{\dagger}$ \\ Beijing University of Aeronautics and Astronautics, 100083 Beijing, China \\ Po-yang Jay Liü, D.W. Zingg ${ }^{\S}$ \\ University of Toronto Institute for Aerospace Studies, Toronto, Canada
}

Keywords: Optimization, Inverse Design, Maximum Endurance Factors

\begin{abstract}
$\underline{\text { Abstract }}$
Numerical optimization of low - Reynolds number, long-endurance airfoils has been investigated with an adjoint Navier Stokes aerodynamics shape optimization method, and an experimentally verified inverse approach. The optimized airfoils are compared with the inverse result and an existing airfoil. The optimized airfoils show significantly increased maximum lift coefficient and endurance factors, together with improved pitching moment and reasonable thickness distributions.
\end{abstract}

\section{$\underline{\text { 1. Introduction }}$}

Long-enthasarbecome one of the new targets in recent R\&D activities for air-vehicles. Examples include the ERAST (Environmental Research and Sensor Technology) project of NASA ${ }^{1}$, and other long-endurance airplane and methods investigations in the world ${ }^{2,3}$. Typical applications of such flights include communication relay, natural environmental protection, disaster survey and even sport airplanes. The mission duration of these so-called "Atmospheric Satellites" varies from 20 to 100 hours.

Computational Fluid Dynamics (CFD), as one of the modern tools, has been used to develop the special airfoils and wings to realize the long-endurance flights. One of the practices includes the design of a low-speed, high-lift, mild-stall, long-endurance wing by applying an inverse method. The results have been well validated by wind-tunnel tests ${ }^{4}$.

\footnotetext{
* Professor.jjhua801@hotmail.com

† Associate Professor. frannyfk@hotmail.com

\$ Graduate Student, jay@oddjob.utias.utoronto.ca.

$\S$ Professor, Senior Member AIAA, http://goldfinger.utias. utoronto.ca/ dwz/.

Copyright $(2003$ by J. Hua, F. M. Kong, and D. W. Zingg. Published by the American Institute of Aeronautics and Astronautics, Inc. with permission.
}

Even though the inverse approaches have been well established and used in aerospace industries for decade, the success of the aerodynamics design still strongly depends on the special expertise in prescribing the reasonable target distributions. Meanwhile, numerical optimization methods, which may reduce the dependence on the designer's experience, have been continuously developed and become closer to engineering applications. One successful approach is a Newton-Krylov algorithm based on the discrete adjoint method ${ }^{5}$. This combines a fast solver for the NavierStokes equations with an efficient gradient calculation and a robust gradient-based optimizer. The NewtonKrylov algorithm has been shown to be roughly thirty times faster than a genetic algorithm ${ }^{6}$ and has been applied to numerous multi-point and multi-objective design problems including the design of high-lift systems ${ }^{7}$.

The purpose of this investigation is to compare the use of the above mentioned optimization procedure, with the inverse design approach in the design of airfoils for long-endurance flight. In addition to providing a high endurance factor, $C_{1}^{3 / 2} / C_{d}$, the airfoil must satisfy certain thickness constraints and have good properties related to the pitching moment coefficient and stall characteristics.

\section{Inverse Design Methods and Result}

\subsection{The requirement for long endurance flight}

A good solution to the long endurance flight is to operate the aircraft at almost maximum lift and lowest cruise speed with engine power just good enough to maintain the altitude and against the wind, so as to reach the minimum fuel consumption and longest mission endurance. This is particularly true when the aircraft is making a stationary circle-route for telecommunication relay. 
Such a flight mission then turns to the challenge to develop new long-endurance airfoils with the following requirements:

- High operational lift coefficient, $\mathrm{Cl}>1$;

- High endurance factor $\mathrm{Cl}^{3 / 2} / \mathrm{Cd}$;

- Very mild stall characteristics for enough safety margins;

- $\quad$ Limited pitching moment coefficient $\mathrm{Cm}$;

- To realize the above features at low Reynolds numbers $\left(\operatorname{Re} \sim 10^{6}\right)$;

- $\quad$ Large relative thickness $\mathrm{T} / \mathrm{C}$.

\subsection{The inverse method and design software}

For designing the airfoil to reach a target pressure distribution, the 2D/3D Transonic Design CFD software NPU-TDTDTD has been used which includes 2D/3D inverse codes, a GA optimizer and a dozen airfoil/wing analysis codes based on FP/Euler + Boundary-Layer methods and Navier Stokes solvers. The inverse solvers of this software are modified based on the "iterative residual correction" principle of Takanashi ${ }^{8}$ and are able to generate new airfoils and wings within a few design iterations with the $\mathrm{Cp}$ converged very satisfactorily to the targets, even when strong discontinuities like shock waves exist. The software has been continuous upgraded and used for a number of 2D/3D designs of supercritical and laminar flow configurations ${ }^{9}$. Fig. 2-1 presents the basic structure of this software.

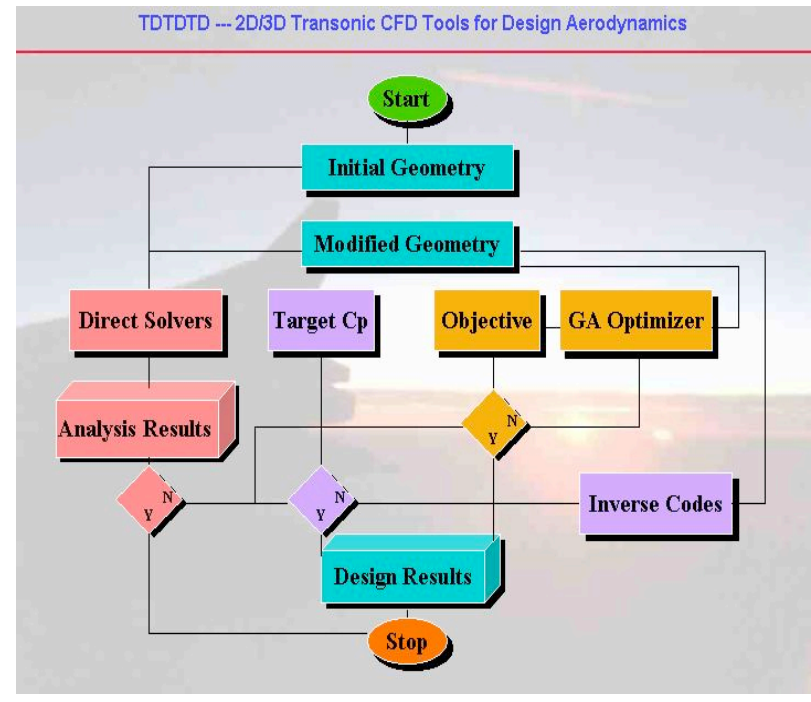

Fig. 2-1. Target Cp on-screen modification

An on-screen target $\mathrm{Cp}$ editing software based on Visual Basic and Windows platform has been developed. The user could easily modify the wanted target $\mathrm{Cp}$ distributions with the mouse, as shown in Fig. 2-2 for a wing section.

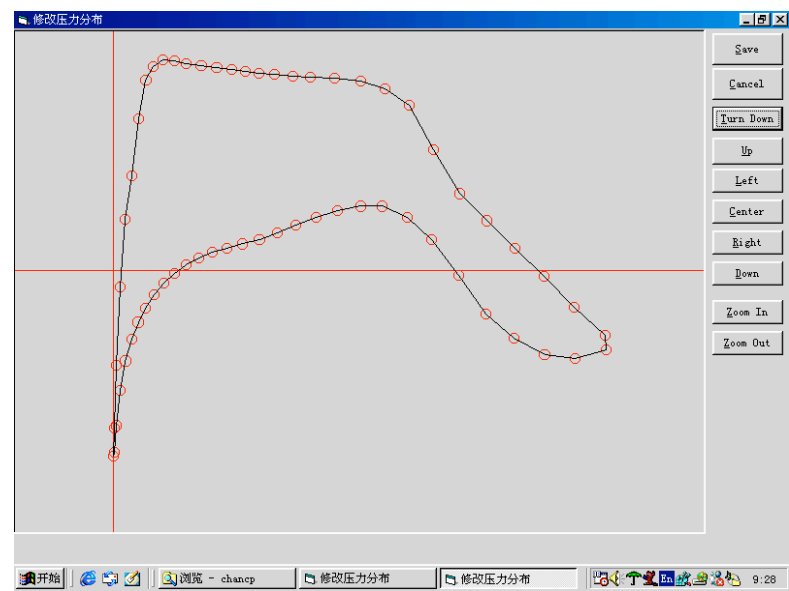

Fig. 2-2. Target $\mathrm{Cp}$ on-screen modification

\subsection{The inverse design of the long-endurance airfoil}

The design point of the low speed long-endurance airfoil for this investigation is chosen as: Mach Number $\mathrm{M}=0.20, \mathrm{Cl}=1.0$ and $\mathrm{Re}=2.0 \mathrm{E} 6$. The new airfoil is supposed to have comparable thickness distributions with the $\mathrm{GA}(\mathrm{W})-1$ profile. The absolute pitching moment $|\mathrm{Cm}|$ is no larger than that of the $\mathrm{GA}(\mathrm{W})-1$ at the design point. The maximum endurance factor should be greatly increased.

Repeated investigation leads to the optimized target $\mathrm{Cp}$ distribution as shown in Fig. 2-3. The target pressure distribution features the following characteristics:

- A round front upper Cp "roof" up to $30 \%$ chord, one key to the mild-stall required;

- A weak Stratford type upper surface pressure recovery for preventing the fast upstream movement of the separation point, which is found important for maintain high lift in low Reynolds numbers;

- Certain amount of front loading in the lower surface for the compensation of the lift and also for resulting in the airfoil to have a nosedown shape, another key to mild-stall.

- Reduced rear loading for the pitch moment control;

From this target $\mathrm{Cp}$, the new airfoil, BUAA-CS2 (Test Case 2) is generated with the above inverse approach. The initial airfoil is NACA-0012 and eight design iterations are used. The good convergence to the target $\mathrm{Cp}$ is shown in Fig. 2-3, too. The new airfoil, plotted together with other optimized results late, has a 
maximum relative thickness $17 \%$, and the trailing edge thickness ratio $0.5 \%$.

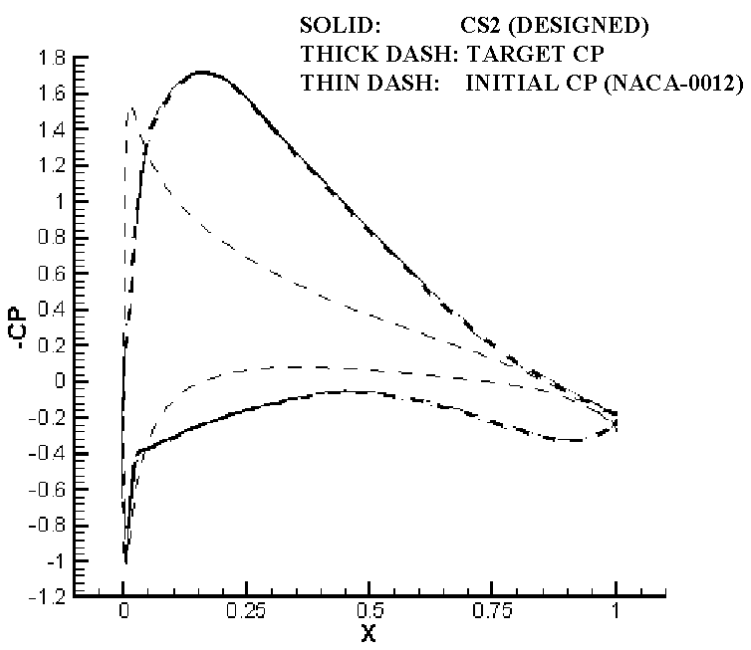

Fig. 2-3. Pressure distributions of the BUAA-CS2 airfoil (initial, target and designed)

\section{Optimization Methods and Results}

\subsection{Newton-Krylov Algorithm for Aerodynamic} Optimization

The present algorithm for aerodynamic optimization is a gradient-based approach in which the discrete adjoint method is used to calculate the gradient efficiently. The solver for the Navier-Stokes equations is a NewtonKrylov algorithm, and the same preconditioned Krylov algorithm is used to solve the linear adjoint problem. A quadratic penalty method is used to cast the constrained optimization problem as an unconstrained problem by incorporating the constraints in the objective function. The resulting unconstrained problem is solved using the BFGS quasi-Newton algorithm coupled with a backtracking line search. One objective function and one gradient calculation are required for each of the design iterations. The optimization terminates when the magnitude of the gradient vector is reduced by four orders of magnitude. One limitation of the present algorithm is that it is restricted to fully turbulent flow, that is, it produces the optimum airfoil shape on the assumption that laminar-turbulent transition occurs at the leading edge on both surfaces. For further details and examples of applications of this aerodynamic optimization algorithm, see [5-7].

\subsection{Long-Endurance Airfoil Design by Optimization}

The CS2 and the GA(W)-1 airfoils are used as the initial contours of the gradient-based optimization approach. The airfoil geometry is described using a set of fifteen B-spline control points. Fig. 3-1 shows the location of the control points for the NACA0012 airfoil. For this study, we perform the optimization with five and seven design variables. The five-design-variable $(5 \mathrm{dv})$ case uses the angle-of-attack and the $5^{\text {th }}, 6^{\text {th }}, 10^{\text {th }}$, and $11^{\text {th }}$ control points as design variables. The sevendesign-variable $(7 \mathrm{dv})$ case uses the $4^{\text {th }}$ and $12^{\text {th }}$ control points as additional design variables. The two extra design variables are located near the tail, which provide freedom for the optimizer to produce a more cambered airfoil. The remaining control points stay unchanged to hold a round leading edge and sharp trailing edge. Note that both cases provide a relatively small number of design variables. We have not examined the effect of increasing this parameter.

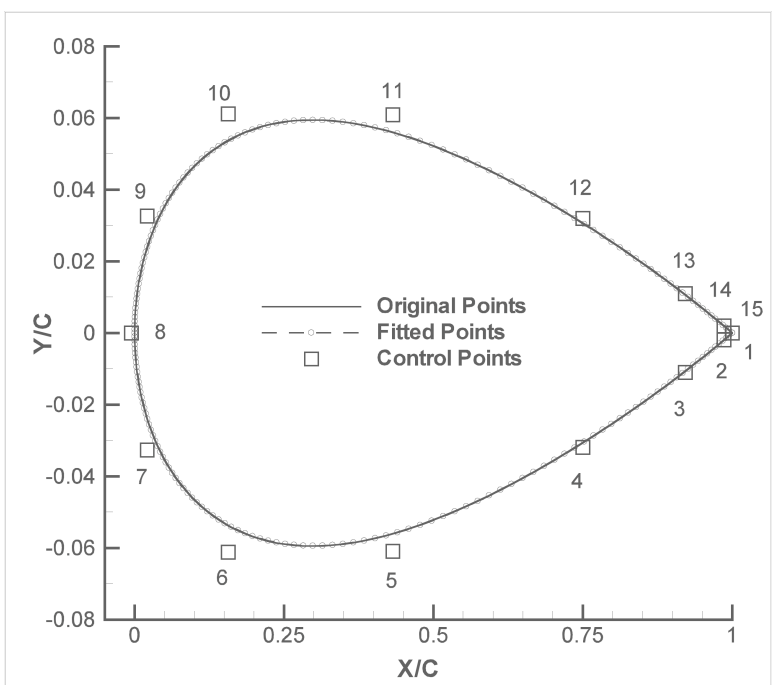

Fig. 3-1. B-spline control points locations

Our objective function, to be minimized, is the reciprocal of the endurance factor. This leads to a single-point optimization in which the maximum endurance factor is maximized, but off-design performance could be poor. As we shall see in the next section, this single-point optimization is effective for this application, probably because the number of design variables is relatively low. The objective function is augmented with penalty terms associated with the constraints. Note that some of the constraints are applied to multiple operating points.

Table 3-1 shows a set of five thickness constraints and their chord locations. If the thickness to chord ratio is below the value specified in the table as t.c. at the given $\mathrm{x} / \mathrm{c}$ location, then a penalty is imposed on the objective function. If the thickness to chord ratio is equal to or greater than that specified, then the objective function is unaffected, and the constraint is described as inactive. The same thickness constraints are imposed on all test 
cases. All but the second constraint are introduced to avoid strange shapes during the optimization iterations; only the second thickness constraint is active once convergence is achieved.

Table 3-1. Thickness constraints

\begin{tabular}{|c|l|l|l|l|l|}
\hline $\mathrm{x} / \mathrm{c}$ & 0.15 & 0.35 & 0.60 & 0.92 & 0.99 \\
\hline t. c. & 0.01 & 0.164 & 0.07 & 0.01 & 0.001 \\
\hline
\end{tabular}

In addition, we consider a third optimization problem specification with additional constraints on the pitching moment. They are applied to the $7 \mathrm{dv}$ case to give a new case designated $7 \mathrm{dv}-\mathrm{Cm}$. At three different angles of attack (AOA), a pitching moment coefficient is specified. If the pitching moment at that angle of attack is less than the specified value, i.e. more negative, then a penalty term is added to the objective function. Otherwise the objective function is unaffected, and the constraint is inactive. The angle of attack and pitching moment coefficient values, which are given in Table 32 , are equal to those for the GA(W)-1 airfoil.

Table 3-2. Moment constraints

\begin{tabular}{|l|c|c|c|}
\hline $\mathrm{AOA}$ & 2 & 5 & 9 \\
\hline $\mathrm{C}_{\mathrm{m}}$ & -0.0949 & -0.0912 & -0.0787 \\
\hline
\end{tabular}

Fig. 3-2 shows the comparison of the above three optimized airfoils, the CS2 obtained with the inverse method, and also the GA(W)-1 airfoil.

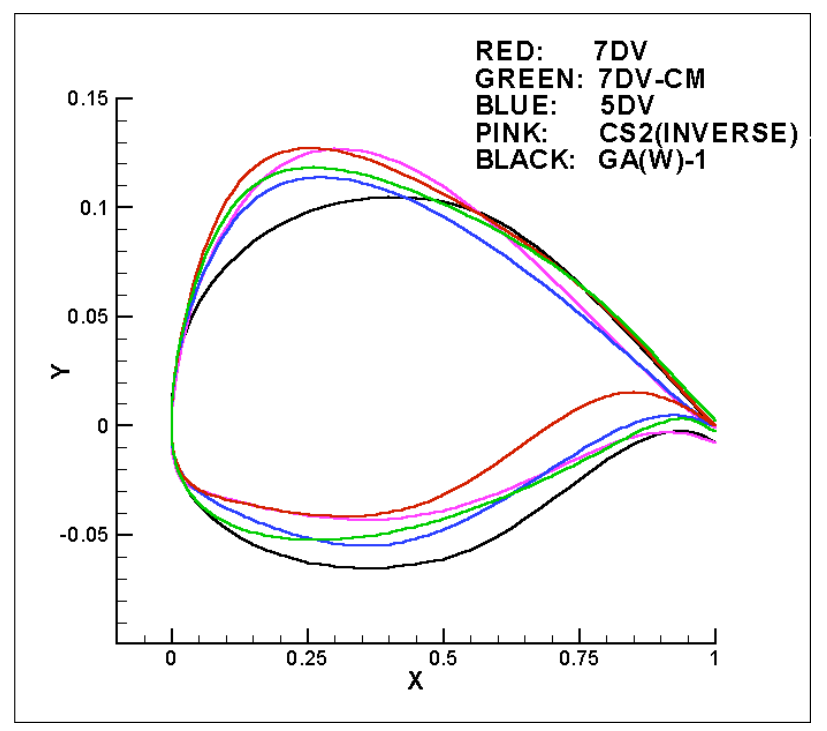

Fig. 3-2. Comparison of the airfoil contours (Y direction amplified)

All the new airfoil contours obtained have $\mathrm{T} / \mathrm{C}$ ratio $17 \%$, but the chord locations of the $\mathrm{T} / \mathrm{C}$ max. move upstream, compared with the $\mathrm{GA}(\mathrm{W})-1$. For example the five-design-variable $(5 \mathrm{dv})$ and the inverse results move about $10 \%$ chord upstream, while the sevendesign-variable $(7 \mathrm{dv} \& 7 \mathrm{dv}-\mathrm{Cm})$ around $20 \%$ chord upstream. The $7 \mathrm{dv}-\mathrm{Cm}$ contour has the rear spar height, that is the $\mathrm{T} / \mathrm{C}$ around $70 \%$ chord, almost the same as the GA(W)-1, while the inverse result CS2 has the next closer value. A higher value of the rear spar height is more favorable for the increase of the wing aspect ratio and the installation of the flap system.

\section{Numerical Analysis and Comparisons of the Airfoils}

\section{4-1 Analysis at Free Transition}

At Reynolds number 2E6, most of the airfoils will have laminar flows over certain chord length, if the wings have only small swept angles and are reasonably manufactured. Then it is important to analyze the aerodynamic characteristics of the new airfoils at free transition conditions. For this task the well-known Euler + Boundary-layer code MSES ${ }^{10}$ is used. The reliability of this code is widely validated, including the wind-tunnel tests for a similar wing ${ }^{4}$.

Fig. 4-1 presents the computed $\mathrm{Cp}$ distributions of all the five airfoils at the design condition $\mathrm{M}=0.20, \mathrm{Cl}=1.0$ and $\mathrm{Re}=2.0 \mathrm{E} 6$.

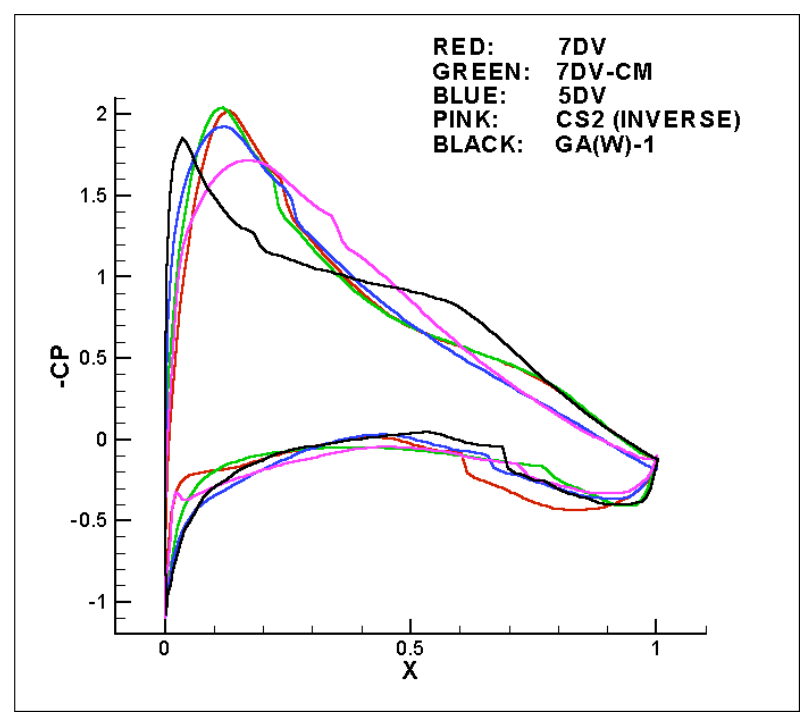

Fig. 4-1. Comparison of the Cp distributions $\mathrm{M}=0.2, \mathrm{Cl}=1.0, \mathrm{Re}=2 \mathrm{E} 6$, Free Transition

The Cp of the GA(W)-1 has a sharp upper surface suction peak close to the leading edge, with the transition point location at about $18 \%$ chord. The inverse result CS2 has a round Cp there, as prescribed, 
and the transition shifted to $35 \%$ chord. All the optimized airfoils, such as $7 \mathrm{dv}, 7 \mathrm{dv}-\mathrm{Cm}$ and $5 \mathrm{dv}$, have stronger suction peaks located chord-wise in between of the $\mathrm{GA}(\mathrm{W})-1$ and $\mathrm{CS} 2$, and the transition locations, too. The $5 \mathrm{dv}$ and CS2 have weaker rear loadings than $\mathrm{GA}(\mathrm{W})-1$, while the $7 \mathrm{dv}$ has the strongest rear loading. The differences of the above $\mathrm{Cp}$ distributions will influence the aerodynamic characteristics of the airfoils, as analyzed while prescribing the inverse target $\mathrm{Cp}$ in Chapter 2.

Fig. 4-2 shows the comparison of the MSES computed $\mathrm{Cl} \sim \square$ curve. At $\mathrm{M}=0.2, \mathrm{Re}=2 \mathrm{E} 6$ and free transition, all the optimized airfoils have larger maximum lift coefficient with the top increase of the $7 \mathrm{dv}$ up to $25 \%$. The inverse designed airfoil CS2 has also improvement over the GA(W)-1, and meanwhile very mild stall characteristics. The $5 \mathrm{dv}$ airfoil, initiated from CS2, has also relatively milder stall than the $7 \mathrm{dv}$ 's started from GA(W)-1.

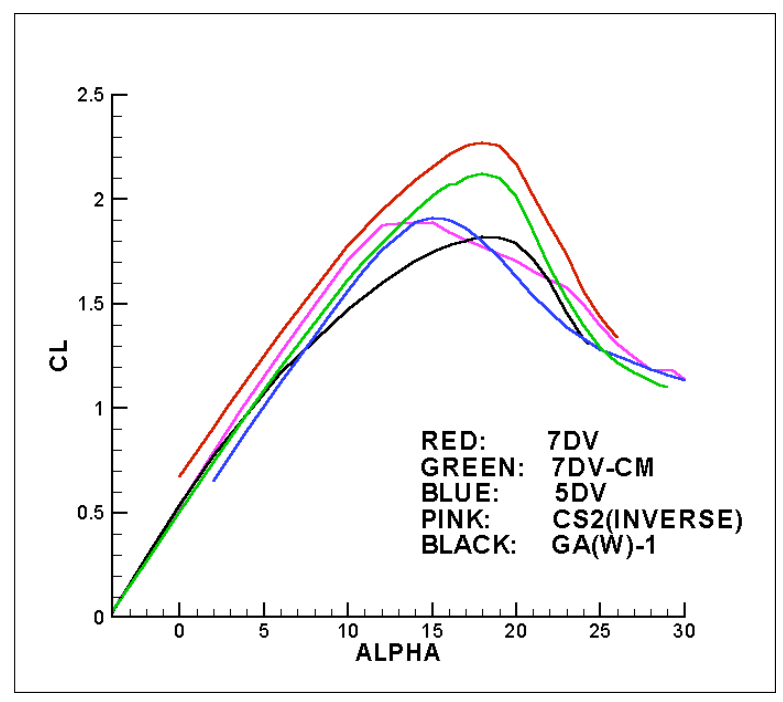

Fig. 4-2. Comparison of the lift curves $\mathrm{M}=0.2, \mathrm{Re}=2 \mathrm{E} 6$, Free Transition

Fig. 4-3 is the comparison of the $\mathrm{Cm}-\mathrm{Cl}$ curves, it could be seen that the optimized airfoil with pitching moment constrains, $7 \mathrm{dv}-\mathrm{Cm}$ in green, has flat and smaller $|\mathrm{Cm}|$ values than $\mathrm{GA}(\mathrm{W})-1$ under $\mathrm{Cl}<1.35$, showing the effectiveness of the constrains. Meanwhile it has the best thickness distribution, see Fig. 3-2, ranking the best airfoil of the optimized ones. The inverse result $\mathrm{CS} 2$ has also comparable $\mathrm{Cm}$ values as the $7 \mathrm{dv}-\mathrm{Cm}$, but the curve is not as flat.

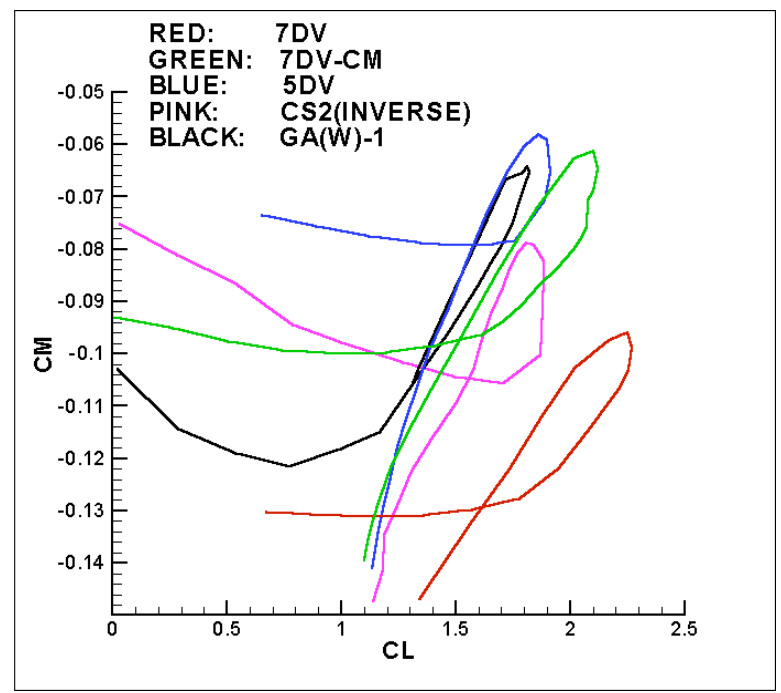

Fig. 4-3. Comparison of the $\mathrm{Cm}-\mathrm{Cl}$ curves $\mathrm{M}=0.2, \mathrm{Re}=2 \mathrm{E} 6$, Free Transition

Fig. 4-4 shows the improvement of the endurance factor. The best candidate airfoil, the $7 \mathrm{dv}-\mathrm{Cm}$, has a $36 \%$ increase in the maximum value over $\mathrm{GA}(\mathrm{W})-1$, and all the new results move the envelops to higher lift coefficients, which promise more loads, higher altitude, lower fuel consumption and longer flight time.

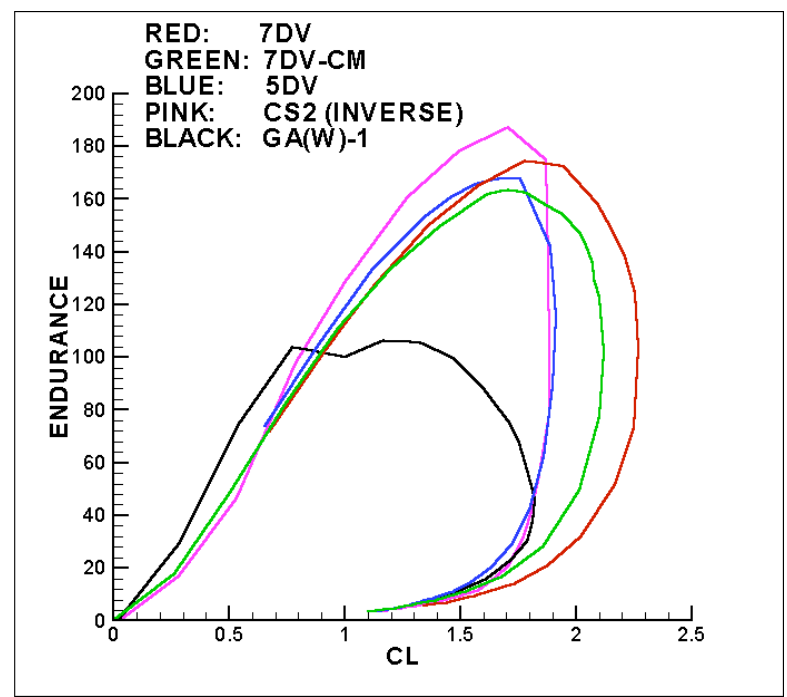

Fig. 4-4. Comparison of the endurance factors $\mathrm{M}=0.2, \mathrm{Re}=2 \mathrm{E} 6$, Free Transition

The reason that the inverse designed airfoil CS2 has the maximum endurance factor in this plot is due to the longer extension of the laminar flow as discussed in the $\mathrm{Cp}$ distributions, while the optimization assumed a fully turbulent flow without laminar regions. Even though, the CS2 airfoil is not necessarily preferable to the $7 \mathrm{dv}$ - 
$\mathrm{Cm}$ airfoil if all factors are taken into account, it is noticed that the possibility to operate the optimized $7 \mathrm{dv}-\mathrm{Cm}$ at higher lift coefficient could certainly provide more payload benefit and safety margins.

\section{4-2 Analysis with Fixed Transition}

In this section, the airfoils are analyzed, again using MSES, with transition specified at $0.07 \%$ chord on both surfaces. While this still does not correspond exactly to the conditions of the optimization, it is closer than the analysis of the preceding section. Furthermore, this analysis is pertinent to an assessment of the new sections, since in practical applications the regions of laminar flow may be reduced as a result of various forms of contamination.

Fig. 4-5 shows the lift curves, the computation failed to converge around 15 degrees when large-scale separation took place, which physically implies the onset of the stall. The higher maximum lift features of the optimized airfoils could be seen clearly which provide more safety margins, as in the free transition cases. The inverse designed CS2 airfoil has meanwhile an earlier but milder stall.

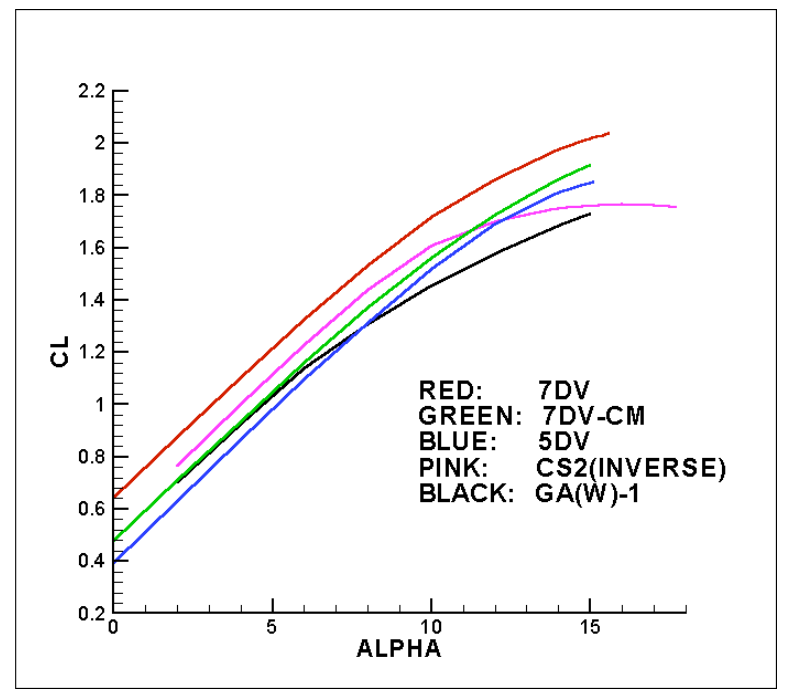

Fig. 4-5. Comparison of the lift curves $\mathrm{M}=0.2, \mathrm{Re}=2 \mathrm{E} 6$, Fixed Transition

The pitching moment characteristics, as shown in Fig. $4-6$, is very similar to the free transition analysis.

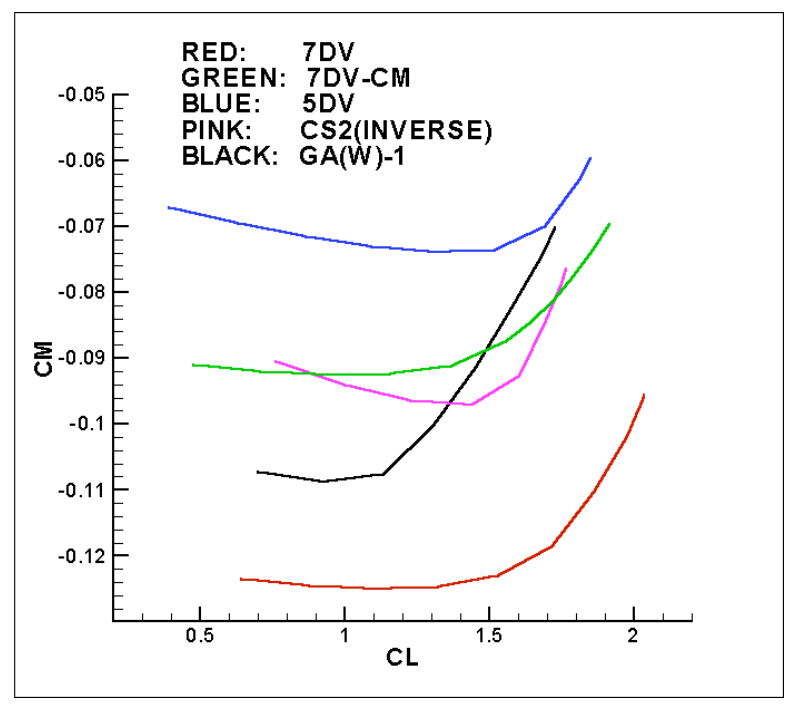

Fig. 4-6. Comparison of the CM-CL curves $\mathrm{M}=0.2, \mathrm{Re}=2 \mathrm{E} 6$, Fixed Transition

The significant improvement in the long endurance factors of the new optimized airfoils is shown very well in Fig. 4-7, under fixed transition conditions. The $7 \mathrm{dv}-\mathrm{Cm}$ airfoil (green line) has $23.7 \%$ increase in the maximum value over the GA(W)-1 contour, and also $9 \%$ over the inverse result. Considering the geometry and the aerodynamic characteristics at both free and fixed transitions, the $7 \mathrm{dv}-\mathrm{Cm}$ airfoil could serve as the most promising result out of this investigation, and it also confirms that the optimization algorithm is working properly.

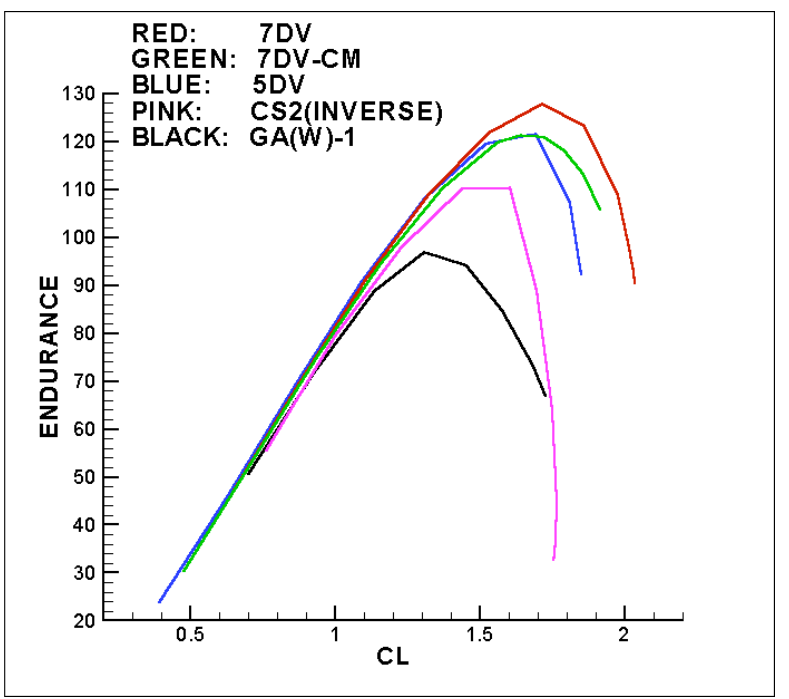

Fig. 4-7. Comparison of the endurance factors $\mathrm{M}=0.2, \mathrm{Re}=2 \mathrm{E} 6$, Fixed Transition 


\section{Conclusions}

- Long endurance flight could be realized by the development of special airfoils with high maximum endurance factors.

- A verified inverse approach is effectively applied in this paper to generate the longendurance airfoil. The expertise of prescribing the target $\mathrm{Cp}$ distribution for high-lift and mild stall becomes important.

- The Newton-Krylov aerodynamic optimization algorithm has been successfully applied to design long-endurance airfoils with reasonable objective functions and constraints investigated. The number of variables will influence the optimized results and the constraint in $\mathrm{Cm}$ is found effective.

- The best result of the optimized airfoils has higher $\mathrm{Cl}_{\max }$, flat and smaller $|\mathrm{Cm}|$, satisfactory thickness distribution and great increase in endurance factors, compared with reference airfoils in free and fixed transition cases. The optimized contours could also provide favorable operation envelops at higher lift coefficient.

- The inverse and optimization approaches produce airfoils of comparable merit. However, the optimization approach requires less expertise on the part of the designer and permits more rapid tailoring of the airfoil characteristics.

\section{$\underline{\text { References }}$}

[1] "ERAST" project, NASA Website.

[2] Selig, M.S., and Guglielmo, J.J., High-Lift Low Reynolds Number Airfoil Design, Journal of Aircraft, Vol. 34, No. 1, 1997.

[3] Bolsunovsky, A.L., Buzoverya, N.P., and Nikolaeva, K.S., Optimization Procedure for Design of High-Lift Airfoils with Specified Stall Behavior, Journal of Advanced Engineering Design, Vol. 40, No. 1, 2000.

[4] Kong, F.M., Hua, J., Xiang, J.W., and Zhang, Z.Y., Investigation of High-Lift, Mild-Stall Wings, ICAS-2002-2.4.2, 2002.

[5] Nemec, M., and Zingg, D.W., An Accurate and Efficient Algorithm for the Navier-Stokes Design Problem, ICAS-2002-1.7.3, 2002

[6] Nemec, M., and Zingg, D.W., Towards Efficient Aerodynamic Shape Optimization Based on the Navier-Stokes Equations, AIAA 2001-2532, 2001.

[7] Nemec, M., and Zingg, D.W., Newton-Krylov Algorithm for Aerodynamic Design using the
Navier-Stokes Equations, AIAA Journal, Vol. 40, No. 6, 2002.

[8] Takanashi, S., An Iterative Procedure for ThreeDimensional Transonic Wing design by the Integral Equation Method, AIAA-85-1595, 1985.

[9] Hua, J., Kong, F.M., Wang, J.J., Zhan, H., Sun, R.Z., Qiu, D., Fu, D.W., Liu, J.C., and Zhang, Z.Y., Recent Examples on Design Aerodynamics for Transport Aircraft, ICAS-2002-2.5.2, 2002.

[10] Drela, M., Newton Solution of Coupled ViscousInviscid Multi-element Airfoil Flow, AIAA 901470, 1990. 